\title{
Consumo de alcohol durante el embarazo
}

\section{Alcohol consumption during pregnancy}

En la historia de la humanidad, el problema de los efectos nocivos del consumo de alcohol en la mujer embarazada ha sido reconocido en diversas ocasiones. El mismo Aristóteles, en el siglo IV a.C., alertaba sobre estos: "Las mujeres que beben alcohol suelen tener hijos taciturnos y lánguidos...". Durante la epidemia, con el consumo de gin en Londres, en 1700, se observó un marcado incremento de la mortalidad fetal e infantil y de neonatos que nacían débiles e hipotónicos.

Sin embargo, las primeras descripciones médicas publicadas de los efectos de la exposición prenatal al alcohol (EPA) se deben a dos pediatras franceses, Jacqueline Rouquette (1957) y Paul Lemoine (1968). ${ }^{1}$ A pesar de esto, la mayoría de los artículos sobre el tema mencionan a Smith y Jones como quienes describieron y definieron por primera vez, en 1973, el "síndrome de alcohol fetal" (SAF). ${ }^{2}$

Esta definición no ha variado sustancialmente hasta la actualidad y abarca 3 aspectos típicos, a saber: dismorfias craneofaciales características, trastornos en el crecimiento pre- y posnatal, y alteraciones del neurodesarrollo y la conducta. Desde ese momento y hasta el presente, se han publicado alrededor de 17000 trabajos científicos que han colaborado en establecer un amplio rango de efectos dependientes de las dosis de alcohol durante el embarazo. A este amplio rango de efectos físicos, neurocognitivos y conductuales relacionados con la EPA se lo ha denominado "espectro de desórdenes fetales del alcohol" (EDFA). El SAF representaría el extremo más grave del EDFA. ${ }^{1-3}$

Se considera que el EDFA es un importante problema de salud pública a nivel mundial, muy poco incluido en la planificación de las políticas sanitarias, en la formación de salud de pre- y posgrado, y en la práctica médicoasistencial de nuestro país. Específicamente, en perinatología y pediatría, es una situación poco reconocida y diagnosticada, a pesar de que de su detección e intervención oportuna depende la calidad de vida de estos niños y su familia. Actualmente, representa la principal causa congénita prevenible de discapacidad intelectual en el mundo occidental. ${ }^{4}$

En el año 2010, el Dr. Sergio Evrard realizó una excelente revisión sobre el tema en Archivos
Argentinos de Pediatría al exponer las características propias del SAF y del EDFA. ${ }^{1}$ En este trabajo, se describieron en profundidad los requisitos básicos para el diagnóstico de cada una de las entidades mencionadas. Estos criterios han sido refinados en publicaciones posteriores, en los que se aclara que es suficiente la corroboración de la tríada compuesta por dimorfismos craneofaciales específicos, retraso en el crecimiento y desórdenes mentales que impactan, fundamentalmente, sobre las funciones ejecutivas ${ }^{2}$ para definir el SAF. ${ }^{3}$

Distintos estudios nacionales han demostrado que los adolescentes comienzan a consumir alcohol en edades tempranas (el $80 \%$ antes de los 15 años) y que los patrones de consumo se han igualado entre los hombres y las mujeres. Si se calcula que el $45 \%$ de los embarazos no son planeados y que entre un $15 \%$ y un $25 \%$ de las mujeres tienen consumos episódicos excesivos o binge drinking, el cual implica beber más de 4 tragos estándares en una ocasión (trago estándar: $14 \mathrm{~g}$ de alcohol, equivalente a un vaso de cerveza, una copa de vino o 1 medida de bebida blanca), ${ }^{3,5}$ se infiere que un porcentaje significativo de gestantes expondrán al feto a cantidades significativas de etanol antes de reconocer su estado de embarazo.

Un importante estudio realizado en la provincia de Santa Fe en 614 mujeres reveló que el 75,2\% había consumido, al menos, un trago de alcohol y que el 15,1\% reconoció haber tenido un evento de consumo episódico excesivo durante la gestación. Asimismo, menos de la mitad de las mujeres suspendió el consumo de alcohol durante el embarazo, aunque la mayoría expresó que lo había disminuido. ${ }^{5}$

En el Servicio de Neonatología del Hospital Misericordia, junto con un grupo de investigadores del Instituto de Investigación Médica Mercedes y Martín Ferreyra, se desarrolló un estudio clínico sobre los diversos efectos fisiológicos de la exposición a dosis no teratogénicas de alcohol durante la gestación, ${ }^{6}$ en continuidad con una serie de experimentos preclínicos realizados sobre el tema. ${ }^{7}$ En este estudio, se establecieron, a través de entrevistas exhaustivas, 3 categorías de EPA: sin consumo o infrecuente, moderado, excesivo y / o binge. Los neonatos fueron estimulados con el aroma de escasas cantidades de alcohol y con el 
aroma a limón (olor nuevo), mientras eran evaluados en términos de respuestas fisiológicas y comportamentales. Lo interesante de este estudio fue que los recién nacidos hijos de madres con consumo binge exhibieron depresión respiratoria y conductas emocionales indicativas de reconocimiento y afinidad hacia la droga, específicamente, cuando eran estimulados con el olor a alcohol, al que fueron expuestos durante la vida fetal. ${ }^{6}$

Asimismo, diversos estudios epidemiológicos han observado que la EPA es un factor de riesgo del síndrome de muerte súbita del lactante (SMSL). ${ }^{8}$ Como información secundaria del estudio de Anunziata y col. (2020), se observó una ausencia de registro de consumo de drogas legales o ilegales durante el embarazo en las historias clínicas de las madres, lo que evidenció la necesidad de realizar una entrevista dirigida para obtener esta información. ${ }^{9}$ El Dr. W. Hay, ex presidente de la Academia Americana de Pediatría, resaltó la necesidad de investigar formas menos consideradas de programación fetal o fetal programming, que incluían la exposición in utero al alcohol y otras sustancias adictivas, que alteraban posteriormente el desarrollo y la integridad del individuo. ${ }^{10}$

En el año 2018, la Secretaría de Políticas Integrales sobre Drogas de la Nación Argentina (SEDRONAR) lanzó una campaña con el lema "Si hay bebé, no bebas", que alertaba que no había cantidades seguras de consumo de alcohol durante el embarazo (véase https: / / www. youtube.com / watch? $\mathrm{v}=\mathrm{mgMc} 0 \mathrm{Jr} X Y \mathrm{fc}_{\mathrm{c}}$ ). Es importante que existan políticas públicas que encaren este problema de manera integral, enfocadas en la prevención comunitaria, en la investigación local y en la capacitación de los equipos de salud. Se cuenta con una serie de herramientas para detectar estos trastornos, que tienen un alto costo sanitario, económico, educativo, individual y social. Es fundamental el trabajo conjunto intersectorial entre salud, educación e investigación y la motivación del equipo de salud perinatal/pediátrico para trabajar activamente en esta problemática.
Mgtr. Luis A. Ahumada, Lic. Florencia Anunziata $y$ Dr. Juan C. Molina ${ }^{b}$

a. Hospital Misericordia Nuevo Siglo, Córdoba, Argentina.

b. Instituto de Investigación Médica Mercedes y Martín Ferreyra, Córdoba, Argentina.

http: / / dx.doi.org/10.5546/ aap.2021.6

Texto completo en inglés:

http:/ / dx.doi.org/10.5546/ aap.2021.eng.6

Cómo citar: Ahumada LA, Anunziata F, Molina JC. Consumo de alcohol durante el embarazo. Arch Argent Pediatr 2021;119(1):6-7.

\section{REFERENCIAS}

1. Evrard SG. Diagnostic Criteria for Fetal Alcohol Syndrome and Fetal Alcohol Spectrum Disorders. Buenos Aires; 2010. http:/ / www.fondation-paul-parquet.com/

2. Hoyme HE, Kalberg WO, Elliott AJ, Blankenship J, et al. Updated Clinical Guidelines for Diagnosing Fetal Alcohol Spectrum Disorders. Pediatrics. 2016; 138(2):e20154256.

3. Williams JF, Smith VC, Committee on substance abuse. Fetal Alcohol Spectrum Disorders. Pediatrics. 2015. 136(5):e1395-406.

4. Tsang TW, Elliott EJ. High global prevalence of alcohol use during pregnancy and fetal alcohol syndrome indicates need for urgent action. Lancet Glob Health. 2017; 5(3):e232-3.

5. López MB, Filiperti VA, Cremonte M. Consumo de alcohol antes y durante la gestación en Argentina : prevalencia y factores de riesgo. Rev Panam Salud Pública. 2015; 37(4/5):211-7.

6. Anunziata F, Macchione AF, Mitrano AS, D'aloisio G, et al. Respiratory and emotional reactivity to ethanol odor in human neonates is dependent upon maternal drinking patterns during pregnancy. Drug Alcohol Depend. 2020; 213:108100.

7. Miranda-Morales RS, D'aloisio G, Anunziata F, Abate P, et al. Fetal Alcohol Programming of Subsequent Alcohol Affinity: A Review Based on Preclinical, Clinical and Epidemiological Studies. Front Behav Neurosci. 2020; 14:33.

8. Bailey BA, Sokol RJ. Prenatal alcohol exposure and miscarriage, stillbirth, preterm delivery, and sudden infant death syndrome. Alcohol Res Health. 2011; 34(1):86-91.

9. Jones TB, Bailey BA, Sokol RJ. Alcohol use in pregnancy: Insights in screening and intervention for the clinician. Clin Obstet Gynecol. 2013; 56(1):114-23.

10. Hay WWJr. American PediatricSociety presidential address 2008: research in early life - benefit and promise. Pediatr Res. 2009; 65(1):117-22. 